\title{
An additional tool towards overcoming absence of specificity of carbon nanostructure-based electrochemical sensors-application to estriol and estradiol detection and distinction
}

\author{
Ivana Cesarino $^{1}$ Ivo A. Hümmelgen ${ }^{2}$
}

Received: 14 May 2015 / Revised: 3 June 2015 / Accepted: 7 June 2015 / Published online: 18 June 2015

(C) Springer-Verlag Berlin Heidelberg 2015

\begin{abstract}
The tristimulus analysis was applied in differential pulse voltammetry experiments to detect and distinguish two substances using a set of three sensors that respond to both substances, aiming at the development of an additional tool to overcome the absence of specificity of the sensor to a single chemical species. The sensors are based on carbon nanostructures (graphene oxide, reduced graphene oxide, and reduced graphene oxide modified with antimony nanoparticles) and are applied to estriol and estradiol detection in water, which constitutes an environmental problem. We show that apart from substance identification, the method can be used to quantify the relative concentration of the substances in a solution.
\end{abstract}

\section{Introduction}

The use of electrochemical sensors is advantageous in several applications due to their high sensitivity associated to the potential low cost of the devices. In most of the cases, however, the signature of the presence of an analyte, an oxidation or reduction peak at a given poten-

Ivo A. Hümmelgen

iah@fisica.ufpr.br

1 Department of Bioprocess and Biotechnology, College of Agricultural Sciences, São Paulo State University, Campus Lageado, 18, Botucatu, SP 603-970, Brazil

2 Departamento de Física, Universidade Federal do Paraná, Caixa Postal 19044, Curitiba, PR 81531-990, Brazil tial, is not specific for a single analyte molecular structure. This is the case, for example, in carbon nanostructure-based sensors, which may present oxidation/reduction peaks for different analytes at nearly the same potential or at potential difference smaller than the full width at half maximum of the peaks, making distinction difficult. For several applications, it may constitute a problem because the practical target may be the detection of the presence or quantification of the concentration, for example, of a hazardous species in a medium containing an intentionally produced substance. In such cases, a hazardous substance cannot be misinterpreted as an inert substance that produces similar sensor response, due to the possibility of disastrous consequences.

The interaction of the analyte and the sensor not only depends on various physico-chemical aspects related to the energy involved in the interaction processes, but it is also dependent on stereochemical aspects, which may impose constraints regarding the diffusivity of an analyte in a porous or layered electrode material and impose difficulties to achieve interaction active sites, for example. As a consequence, even in cases where different analytes lead to current peaks at similar oxidation/ reduction potentials, it is expected that these different analytes lead to different sensitivities. The set of sensitivities of a set of sensors can then also be used as an additional molecular species identification tool, as will be demonstrated later.

\section{Theory}

If we consider a set of three sensors that produce a response in the presence of an analyte, assuming again that these sensors show a linear current intensity response on analyte concentration and absence of 
response when the analyte is absent, the current responses of the three sensors can be written as follows:

$\left\{\begin{array}{l}I_{1}=\alpha[A] \\ I_{2}=\beta[A] \\ I_{3}=\gamma[A]\end{array}\right.$

where the subscript $i$ of the current $I_{i}$ indicates the sensor ( $i=1,2$ or 3$),[A]$ denotes the concentration of analyte A and $\alpha, \beta$, and $\gamma$ are the sensitivities of the three sensors to analyte A. Adopting a procedure similar to that used in the optometric tristimulus analysis to construct chromaticity coordinates [1], which was recently adapted to electronic sensors applications (volatiles detection) in the study of fungus infection in melons and monitoring of the ripening stage of tomatoes $[2,3]$, we can then define the following:

$$
\left\{\begin{array}{l}
i_{1}=\frac{I_{1}}{I_{1}+I_{2}+I_{3}}=\frac{\alpha}{\alpha+\beta+\gamma} \\
i_{2}=\frac{I_{2}}{I_{1}+I_{2}+I_{3}}=\frac{\beta}{\alpha+\beta+\gamma} \\
i_{3}=\frac{I_{3}}{I_{1}+I_{2}+I_{3}}=\frac{\gamma}{\alpha+\beta+\gamma}
\end{array}\right.
$$

These $i$ values essentially can be geometrically seen as the coordinates of the point where the vector given by $I_{1} \hat{x}+I_{2} \hat{y}+I_{3} \hat{z}$ ( $\hat{x}, \hat{y}$, and $\hat{z}$ are orthogonal to each other) crosses the unitary plan given by $i_{1}+i_{2}+i_{3}=1$. These coordinates represent the set of sensitivities of the three sensors to analyte A and can be used as a signature of analyte A, complementing the information of the current intensity, which can be used for concentration determination. Geometrically, it can be represented as shown in Fig. 1. It is important that this strategy makes the coordinates independent of the concentration of the analyte because the concentration only affects the magnitude of the vector $I_{1} \hat{x}+I_{2} \hat{y}+I_{3} \hat{z}$, but not the coordinates of the point where it crosses the unitary plane. Considering that $i_{1}+i_{2}+i_{3}=1$, two of these three coordinates are enough to univocally determine the position because the third one is then also defined through the summation. For this reason, the projection of the point where the vector crosses the

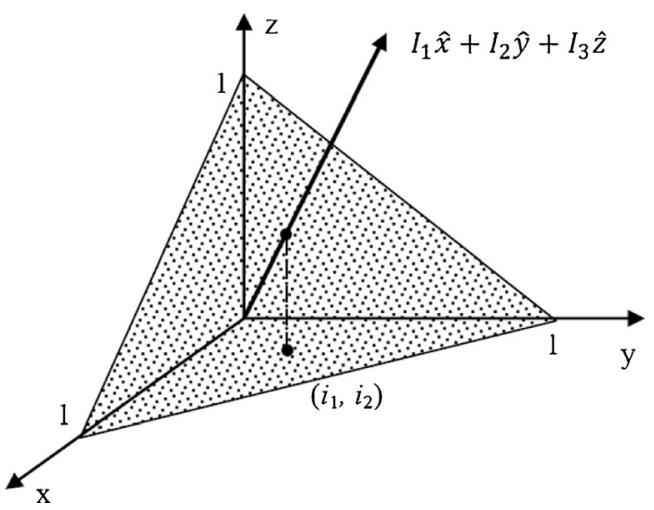

Fig. 1 Geometrical representation of the vector constructed with the current intensity responses of the three sensors (Eq. (1)) crossing the unitary plain in a point that leads to coordinates $\left(i_{1}, i_{2}\right)$ (Eq. (2)) when projected on plane $x y$

unitary plan on plane $x y$ (or alternatively $y z$ or $z x$ ) results in two coordinates $\left(i_{1}, i_{2}\right)$ [or alternatively $\left.\left(i_{2}, i_{3}\right)\left(i_{3}, i_{1}\right)\right]$ that represent the set of responses of the three sensors, with the advantage of being a more usual bidimensional orthogonal axis representation, as illustrated in Fig. 1.

A slightly more complex situation occurs when there are two compounds (A and B) simultaneously present in a solution. In terms of relative concentrations, it can be written that $[A]_{\mathrm{r}}+[B]_{r}=1$. In this case, the contribution of $\mathrm{A}$ to the oxidation current is given by

$$
\left\{\begin{array}{l}
I_{1}^{A}=\alpha^{\prime}[A]_{r} \\
I_{2}^{A}=\beta^{\prime}[A]_{r} \\
I_{3}^{A}=\gamma^{\prime}[A]_{r}
\end{array}\right.
$$

and the contribution of $\mathrm{B}$ to the oxidation current is given by

$$
\left\{\begin{array}{c}
I_{1}^{B}=\alpha^{\prime \prime}[B]_{\mathrm{r}}=\alpha^{\prime \prime}\left(1-[A]_{\mathrm{r}}\right) \\
I_{2}^{B}=\beta^{\prime \prime}[B]_{\mathrm{r}}=\beta^{\prime \prime}\left(1-[A]_{\mathrm{r}}\right) . \\
I_{3}^{B}=\gamma^{\prime \prime}[B]_{\mathrm{r}}=\gamma^{\prime \prime}\left(1-[A]_{\mathrm{r}}\right)
\end{array}\right.
$$

The current in the sensors $(i=1,2$, or 3$)$ will be $I_{\mathrm{i}}=$ $I_{\mathrm{i}}^{A}+I_{\mathrm{i}}^{B}$ and the coordinates $i_{\mathrm{i}}$ can be calculated as

$$
\left\{\begin{array}{l}
i_{1}=\frac{I_{1}^{A}+I_{1}^{B}}{I_{1}^{A}+I_{1}^{B}+I_{2}^{A}+I_{2}^{B}+I_{3}^{A}+I_{3}^{B}}=\frac{\alpha^{\prime}+\left(\alpha^{\prime}-\alpha^{\prime \prime}\right)[A]}{\alpha^{\prime \prime}+\beta^{\prime \prime}+\gamma^{\prime \prime}+\left\{\left(\alpha^{\prime}-\alpha^{\prime \prime}\right)+\left(\beta^{\prime}-\beta^{\prime \prime}\right)+\left(\gamma^{\prime}-\gamma^{\prime \prime}\right)\right\}[A]}=\alpha^{\prime}+\left(\alpha^{\prime}-\alpha^{\prime \prime}\right)[A] \\
i_{2}=\frac{\beta_{2}^{A}+I_{2}^{B}}{I_{1}^{A}+I_{1}^{B}+I_{2}^{A}+I_{2}^{B}+I_{3}^{A}+I_{3}^{B}}=\frac{\left.\beta^{\prime}-\beta^{\prime \prime}\right)[A]}{\alpha^{\prime \prime}+\beta^{\prime \prime}+\gamma^{\prime \prime}+\left\{\left(\alpha^{\prime}-\alpha^{\prime \prime}\right)+\left(\beta^{\prime}-\beta^{\prime \prime}\right)+\left(\gamma^{\prime}-\gamma^{\prime \prime}\right)\right\}[A]}=\beta^{\prime}+\left(\beta^{\prime}-\beta^{\prime \prime}\right)[A] . \\
i_{3}=\frac{\gamma_{3}^{A}+I_{3}^{B}}{I_{1}^{A}+I_{1}^{B}+I_{2}^{A}+I_{2}^{B}+I_{3}^{A}+I_{3}^{B}}=\frac{\left.\gamma^{\prime}-\gamma^{\prime \prime}\right)[A]}{\alpha^{\prime \prime}+\beta^{\prime \prime}+\gamma^{\prime \prime}+\left\{\left(\alpha^{\prime}-\alpha^{\prime \prime}\right)+\left(\beta^{\prime}-\beta^{\prime \prime}\right)+\left(\gamma^{\prime}-\gamma^{\prime \prime}\right)\right\}[A]}=\gamma^{\prime}+\left(\gamma^{\prime}-\gamma^{\prime \prime}\right)[A]
\end{array}\right.
$$


<smiles>C[C@]12CC[C@H]3c4ccc(O)cc4CC[C@H]3[C@@H]1C[C@@H](O)[C@H]2O</smiles>

b<smiles>C[C@]12CC[C@@H]3c4ccc(O)cc4CC[C@H]3[C@@H]1CC[C@@H]2O</smiles>

Fig. 2 Structures of $\mathbf{a}$ estriol and $\mathbf{b}$ estradiol

In the limit, when $[A] \rightarrow 0$, the coordinates equal those of compound $\mathrm{B}$ and when $[A] \rightarrow 1$ those of compound $\mathrm{A}$, as expected for pure substances. The coordinates from any mixture of $\mathrm{A}$ and $\mathrm{B}$ are located in the straight line that connects the coordinates of the pure substances. The ratio $[A] /[B]$ of the mixture in the solution equals the ratio of the straight line segments from the coordinates of the mixture to the coordinates of pure $\mathrm{A}$ and of pure $\mathrm{B}$, respectively. For this reason, it can be used to determine the composition of the mixture of $\mathrm{A}$ and $\mathrm{B}$ in the solution.

The method allows the distinction of A from B, provided that one of the following three conditions given below is obeyed:

$\frac{\alpha}{\alpha^{\prime}} \neq \frac{\beta}{\beta^{\prime}}$ or $\frac{\alpha}{\alpha^{\prime}} \neq \frac{\gamma}{\gamma^{\prime}}$ or $\frac{\gamma}{\gamma^{\prime}} \neq \frac{\beta}{\beta^{\prime}}$.

Better distinction is obtained using the parameters that lead to the bigger difference in the inequalities (Eq. (6)).

\section{Estriol and estradiol}

Estriol and estradiol are bioidentical hormones known as endocrine disruptors and found as a pollutant in environmental waters. These endocrine disruptors can persist after water purification treatments and even at low concentrations can cause undesired biological effects [4]. Therefore, the monitoring of endocrine disruptors in the aquatic environment is progressively becoming a priority for government and regulatory agencies as well as for the general public.

Different physico-chemical principles have been successfully used for estriol and estradiol detection, like adsorptive
Table 1 Peak oxidation current intensity measured in the presence of a $50.0 \mu \mathrm{mol} \mathrm{L}{ }^{-1}$ solution either of estriol or estradiol in case of the three carbon nanostructure (GO, rGO, or GC/rGO-SbNPs) based sensors and the corresponding values of $i$

\begin{tabular}{clll}
\hline & \multicolumn{2}{l}{ Electrode } & \\
\cline { 2 - 4 } & $\mathrm{GC} / \mathrm{GO}$ & $\mathrm{GC} / \mathrm{rGO}$ & $\mathrm{GC} / \mathrm{rGO} / \mathrm{SbNPs}$ \\
\hline$I(\mu \mathrm{A})$, estriol & 0.245 & 0.331 & 0.471 \\
$i$, estriol & 0.234 & 0.316 & 0.450 \\
$I(\mu \mathrm{A})$, estradiol & 0.550 & 0.848 & 1.209 \\
$i$, estradiol & 0.211 & 0.325 & 0.464 \\
\hline
\end{tabular}

cathodic stripping voltammetry [5], single-sweep polarography [6], surfactant-modified voltammetry using carbon paste electrodes [7], electrocatalytic oxidation at nickel-modified glassy carbon electrode [8], and voltammetry using borondoped diamond electrodes [9]. Graphene oxide (GO), reduced graphene oxide (rGO), and rGO modified with antimony nanoparticles (rGO-SbNPs) are attractive materials for the detection of estriol and estradiol because they show a pronounced electrochemical oxidation peak, showing high sensitivity and presenting constant sensitivity over a wide range of estriol and estradiol (see Fig. 2) concentration [10]. However, an applicability limitation is imposed by the absence of specificity, since both substances show oxidation peaks centered at only slightly different oxidation potential. In this contribution, we apply the above described alternative strategy, based on a tristimulus analysis, to the detection of estriol or estradiol, which would also allow estimating their relative concentration if they are mixed in solution.

\section{Materials and methods}

\section{Apparatus and procedures}

Differential pulse voltammetry (DPV) was performed using a PGSTAT30 Autolab electrochemical system (Eco Chemie,
Fig. 3. Voltammograms of the carbon nanostructure-based sensors in the presence of either a $50.0 \mu \mathrm{mol} \mathrm{L}{ }^{-1}$ solution of: a estriol or $\mathbf{b}$ estradiol (adapted from Ref. [6])
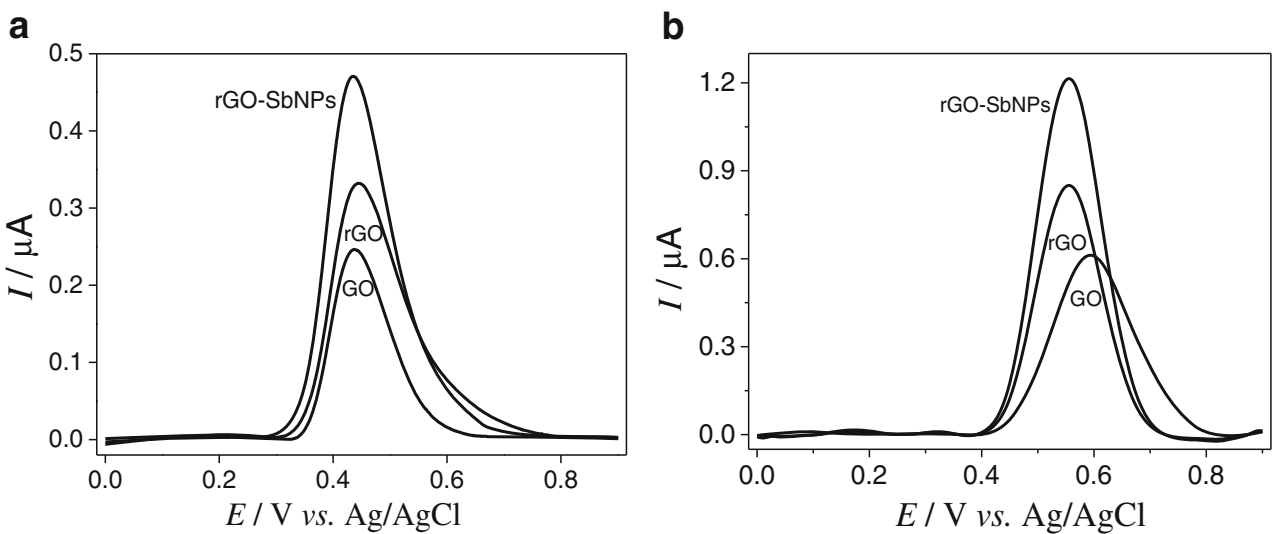
Utrecht, Netherlands) equipped with GPES software (Eco Chemie, Utrecht, Netherlands) and a conventional threeelectrode electrochemical system: either bare glassy carbon (GC), GC/GO, GG/rGO, or GC/rGO-SbNPs as a working electrode (in all cases diameter of $3 \mathrm{~mm}$ ); $\mathrm{Ag} / \mathrm{AgCl} / \mathrm{KCl}$ $\left(3.0 \mathrm{~mol} \mathrm{~L}^{-1}\right)$ as a reference electrode; and a Pt plate as an auxiliary electrode. DPV measurements were recorded at $10 \mathrm{mV} \mathrm{s}^{-1}$, pulse amplitude of $100 \mathrm{mV}$, and a step potential of $2 \mathrm{mV}$ in a $0.1 \mathrm{~mol} \mathrm{~L}^{-1}$ phosphate buffer solution (PBS) $\mathrm{pH} 9.0$, containing $50.0 \mu \mathrm{mol} \mathrm{L}-1$ of estriol or estradiol standards, at a $25^{\circ} \mathrm{C}$.

\section{Chemicals and solutions}

All solutions were prepared with purified water (resistivity $\geq 18 \mathrm{M} \Omega \mathrm{cm}$, Barnsted Nanopure system, Thermo Scientific, USA). All reagents used in this study were of analytical grade and were used without further purification. Graphite powder of 1-2- $\mu \mathrm{m}$ particle size, antimony chloride $\left(\mathrm{SbCl}_{3}\right)$, estradiol, and estriol were purchased from Sigma-Aldrich (Germany).

\section{Synthesis of the GO, rGO, and rGO-SbNPs composite}

The graphene oxide (GO) was prepared following an improved Hummers' method [11]: $10 \mathrm{~g}$ of graphite and $10 \mathrm{~g}$ of $\mathrm{NaNO}_{3}$ were mixed with $450 \mathrm{~mL}$ of $\mathrm{H}_{2} \mathrm{SO}_{4}(98 \%)$ in a $2500-\mathrm{mL}$ flask. The mixture was stirred for $30 \mathrm{~min}$ in an ice bath, followed by the addition of $60 \mathrm{~g}$ of $\mathrm{KMnO}_{4}$ under vigorous stirring. In the sequence, the reaction mixture was held under refrigeration at $4{ }^{\circ} \mathrm{C}$ for $24 \mathrm{~h}$. Next, the mixture was stirred at $35^{\circ} \mathrm{C}$ followed by the addition of $450 \mathrm{~mL}$ of $\mathrm{H}_{2} \mathrm{O}$, also under vigorous stirring. After that, the reaction temperature was abruptly raised to $98{ }^{\circ} \mathrm{C}$ and $450 \mathrm{~mL}$ of $\mathrm{H}_{2} \mathrm{O}$ was added, followed by the addition of $300 \mathrm{~mL}$ of $30 \% \mathrm{H}_{2} \mathrm{O}_{2}$ aqueous solution. The reaction mixture was again held under refrigeration at $4{ }^{\circ} \mathrm{C}$ for $24 \mathrm{~h}$. The obtained GO suspension was centrifuged for $30 \mathrm{~min}$ at $8000 \mathrm{rpm}$ and washed several times with $5 \% \mathrm{HCl}$ and distilled water until the $\mathrm{pH}$ of the filtrate was neutral. Finally, the GO obtained was lyophilizedover $24 \mathrm{~h}$.

Table 2 Peak oxidation current intensity, determined taking the current at $\mathrm{E}=0.5 \mathrm{~V}$, measured in the presence of a $50.0 \mu \mathrm{mol} \mathrm{L}{ }^{-1}$ solution of estradiol in case of the three carbon nanostructure (GO, rGO, or GC/rGOSbNPs) based sensors and the corresponding values of $i$

\begin{tabular}{clll}
\hline & \multicolumn{2}{l}{ Electrode } & \\
\cline { 2 - 4 } & $\mathrm{GC} / \mathrm{GO}$ & $\mathrm{GC} / \mathrm{rGO}$ & $\mathrm{GC} / \mathrm{rGO}-\mathrm{SbNPs}$ \\
\hline$I(\mu \mathrm{A})$, estriol & 0.147 & 0.240 & 0.307 \\
$i$, estriol & 0.212 & 0.346 & 0.442 \\
$I(\mu \mathrm{A})$, estradiol & 0.251 & 0.537 & 0.801 \\
$i$, estradiol & 0.158 & 0.338 & 0.504 \\
\hline
\end{tabular}

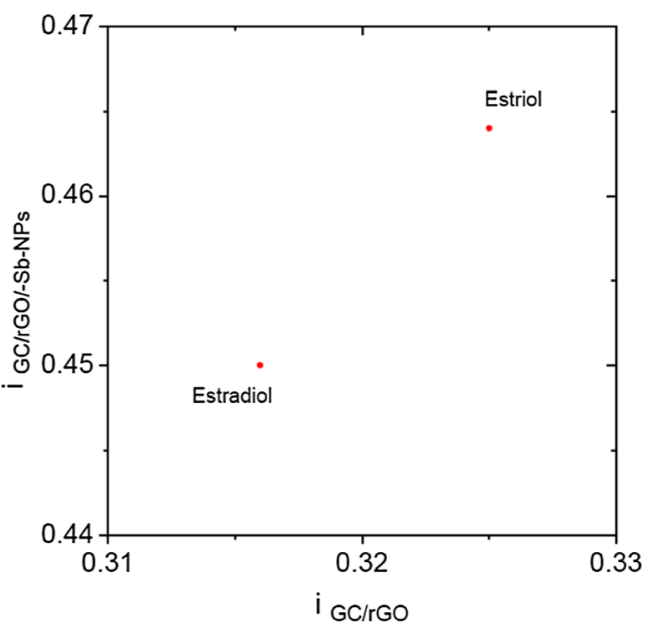

Fig. 4 Plot of the $i_{\mathrm{GC} / \mathrm{rGO}-\mathrm{SbNPs}}$ versus $i_{\mathrm{GC} / \mathrm{rGO}}$ coordinates for a $50.0 \mu \mathrm{mol} \mathrm{L}{ }^{-1}$ solution of estriol and estradiol

The rGO-SbNPs composite was prepared using sodium dodecyl sulfate (SDS) as a surfactant. A suspension containing a 10:4 GO/SDS $w / w$ ratio was prepared in $15 \mathrm{~mL}$ ethanol (pure grade) and sonicated for $20 \mathrm{~min}$. An excess of sodium borohydride was added and this suspension was sonicated for additional $20 \mathrm{~min}$. A solution containing $40 \% \mathrm{SbCl}_{3}$ in relation to the GO amount was slowly dropped into the rGO suspension under constant stirring. After completing the reaction, the rGO-SbNPs composite was dispersed using an ultrasonic probe for $1 \mathrm{~h}$. The obtained rGO-SbNPs were centrifuged for $10 \mathrm{~min}$ at $3000 \mathrm{rpm}$ and washed several times with distilled water until the solution became colorless. After that, $5 \mathrm{~mL}$ of ethanol (pure grade) was added the rGO-SbNPs were

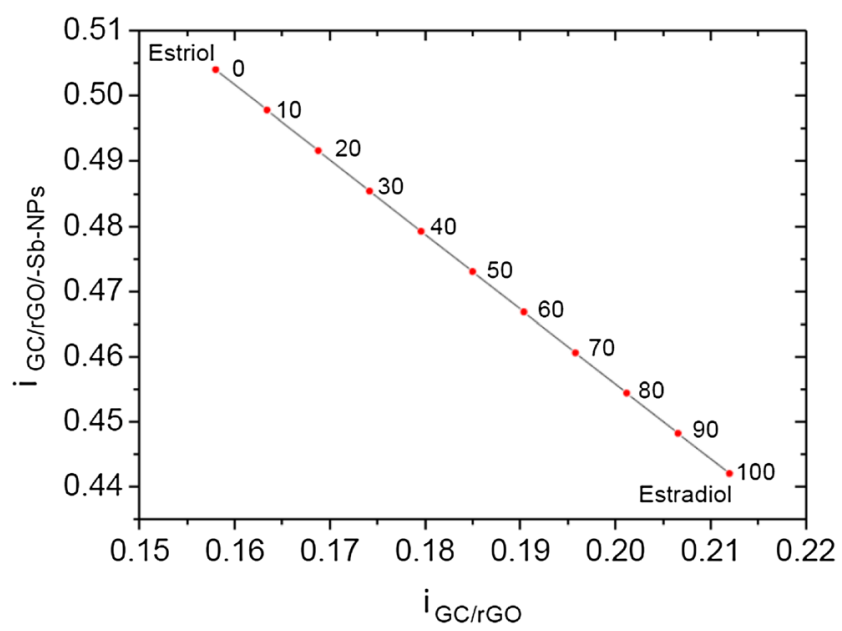

Fig. 5 Plot of the $i_{\mathrm{GC} / \mathrm{rGO}-\mathrm{SbNPs}}$ versus $i_{\mathrm{GC} / \mathrm{GO}}$ coordinates for a $50.0 \mu \mathrm{mol} \mathrm{L}{ }^{-1}$ solution of estriol and estradiol, determined taking the current at $E=0.5 \mathrm{~V}$. The line between the pure estriol and pure estradiol coordinates determines the position of the coordinates of solutions containing both estriol and estradiol. Dots indicate coordinates for the given estradiol percentage 
dried at $60{ }^{\circ} \mathrm{C}$. The rGO synthesis of was also made as described above, except by the addition $\mathrm{SbCl}_{3}$.

\section{Electrodes preparation}

The GC electrode surface was polished with $0.3-\mu \mathrm{m}$ alumina slurries, rinsed thoroughly with double-distilled water, sonicated for $5 \mathrm{~min}$ in ethanol and $5 \mathrm{~min}$ in water, and dried in air prior to modification. A $10-\mu \mathrm{L}$ aliquot of $0.3 \mathrm{mg}$ of $\mathrm{GO}$, rGO, or rGO-SbNPs in 1.0-mL water suspension dispersed using ultrasonic stirring for $20 \mathrm{~min}$ was dropped onto the GC electrode surface and dried at room temperature.

\section{Results and discussion}

The rGO-SbNPs composite was previously FEG-SEMmicroscopy and electrochemically characterized, indicating that the GO was reduced and that the $\mathrm{rGO}$ was modified with the $\mathrm{Sb}$ nanoparticles [10].

The peak oxidation current intensities measured for the three carbon nanostructure (GO, rGO, or GC/rGO-SbNPs) based sensors in the presence of a $50.0 \mu \mathrm{mol} \mathrm{L}{ }^{-1}$ solution of estriol (Fig. 3a) and estradiol (Fig. 3b) were taken from Ref. [10] and are given in Table 1. These tables also contain the values of $i_{\mathrm{GO}}, i_{\mathrm{rGO}}$, and $i_{\mathrm{GC} / \mathrm{rGO}-S b N P s}$ calculated using Eq. (2). It is important to notice that the peak maxima occur at different oxidation potential (approximately $+0.45 \mathrm{~V}$ for estriol and $+0.55 \mathrm{~V}$ for estradiol) and that we simply used their peak intensity values disregarding the corresponding peak potential.

When two of the coordinates calculated using Eq. (2) (see Table 2) are plotted for the estriol and estradiol data (Fig. 3), it is possible to easily distinguish their corresponding coordinates, which means that the two compound can be easily distinguished, as shown in Fig. 4.

In a hypothetical case of a solution containing both estriol and estradiol, the measured current will be a convolution of the curves due to the two compounds in the solution and the corresponding peaks may not be so well defined. But even in this case, assuming independent responses, one can take the current values at an intermediate voltage value between the two oxidation peak voltages, for example, $E=0.5 \mathrm{~V}$ to compute the coordinates given in Table 2, as shown in Fig. 5. If both estriol and estradiol are present in solution, the coordinates will be located in the straight line that connects the coordinates of both pure substances and the relative distance to one of the pure substances, in this case estriol, can be used to directly determine the concentration of the other one, in this case estradiol.

It must be pointed out that the assumption of independent responses is in principle applicable to diffusion-controlled processes with no chemical reactions coupled to the electron transfer process and in the absence of cross-reactions. Essentially, it is necessary to ensure that the product of a first oxidation process adsorbed on the electrode surface does not affect a second oxidation process in a way that it differs from that recorded at the bare electrode. Considering the specific case of estradiol and estriol, the electrochemical detection of these analytes are accompanied by their adsorption or their oxidation products. However, as the estriol and estradiol oxidation potentials are very close, their oxidation occurs practically concurrently; thus, one process is not expected to significantly interfere on the other at reasonably low concentrations and the electrode surface is poisoned only at higher potentials after both oxidation process. A pre-treatment cleaning potential of $-1.0 \mathrm{~V}$ for $60 \mathrm{~s}$ should be applied after each DPV experiments to recover the original response.

It is important to mention that in some cases, the oxidation peak for a given substance may be slightly displaced in the potential scale when the concentration is modified. This displacement will lead to a deviation in the current value if data for all concentrations are taken at the same potential, introducing an error in the determined relative concentration in case of mixtures. However, if a current peak shows severe potential displacements as the concentration of a pure substance changes, this method will lead to large errors and corrections in the determination of mixture relative concentrations will be necessary.

\section{Conclusion}

We showed that the tristimulus analysis, originally developed for the quantification of colors through chromaticity coordinates, can be adapted to be used in the treatment of responses of a set of three electrochemical sensors. We used the current of three sensors recorded in differential pulse voltammetry experiments as a set of inputs to calculate the tristimulus coordinates. We applied the procedure to estriol and estradiol, showing that it is possible to distinguish them, even if current at a common potential for both sets of experiments and that does not match the current peak value is used. We also showed that in a mixture, this procedure can be used to determine the relative concentration of the detected chemical species. The procedure is in principle of general applicability to electrochemical sensors, provided that the sensitivities of these sensors to one of the detected chemical species are not simply simultaneously multiple (with the same multiplication factor) of the sensitivities to the other one. The developed procedure can be used as an additional tool to overcome the absence of specificity commonly observed in electrochemical sensors.

Acknowledgments The authors would like to thank CNPq and FAPE SP (2015/00821-0) for research grants. 


\section{References}

1. Wyszecki G, Stiles WS (2000) Color science-concepts and methods, quantitative data and formulae. Wiley, New York

2. Greenshields MWCC, Mamo MA, Coville NJ, Spina AP, Rosso DF, Destro JG, Porsani MV, Bozza A, Pimentel IC, Hümmelgen IA (2013) Tristimulus mathematical treatment application for monitoring fungi infestation evolution in melon using the electrical response of carbon nanostructure-polymer composite based sensors. Sensors Actuators B Chem 188:378-384

3. Greenshields MWCC, Cunha BB, Mamo MA, Coville NJ, Hümmelgen IA (2015) Non-destructive tomato ripeness determination using carbon nanostructure based chemical sensors. Application of tristimulus and principal component analysis methods. Int J Electroactive Mater 3:10-14

4. Wenzel A, Müller J, Ternes T (2003) Study on endocrine disrupters in drinking water. Schmallenberg and Wiesbaden, Germany

5. Hu S, He Q, Zhao Z (1992) Determination of trace amounts of estriol and estradiol by adsorptive cathodic stripping voltammetry. Analyst 117:181-184
6. Hu S, He Q, Zhao Z (1992) Determination of estradiol and estriol by single-sweep polarography. Anal Chim Acta 259: 305

7. Wu K, He Q, Hu S (2002) Voltammetric determination of estrogens based on the enhancement effect of surfactant at carbon paste electrode. Wuhan Univ J Nat Sci 7:463-469

8. Muna GC, Kaylor A, Jaskowski B, Sirham LR, Kelley CT (2011) Electrocatalytic oxidation of estrogenic phenolic compounds at a nickel-modified glassy carbon electrode. Electroanalysis 23:29152924

9. Brocenschi RF, Rocha-Filho SR, Biaggio SR, Bocchi N (2014) DPV and SWV determination of estrone using a cathodically pretreated boron-doped diamond electrode. Electroanalysis 26: 1588-1597

10. Cesarino I, Cincotto FH, Machado SAS (2015) A synergistic combination of reduced graphene oxide and antimony nanoparticles for estriol hormone detection. Sensors Actuators B Chem 210:453-459

11. Hummers WS, Offeman RE (1958) Preparation of graphitic oxide. J Am Chem Soc 80:1339-1339 